\title{
A comparative study of the epidemiology of Treponemal Infection in the Volta Region of Ghana: A Five-Year Multisite Parallel Population- Based Analysis vis-à-vis the Sentinel Survey
}

\author{
Sylvester Yao Lokpo ( $\square$ sylvesteryao34@gmail.com ) \\ University of Health and Allied Sciences https://orcid.org/0000-0002-1628-0735 \\ Ellis Owusu-Dabo \\ Kwame Nkrumah University of Science and Technology \\ John Gameli Deku \\ University of Health and Allied Sciences \\ Verner Ndudiri Orish \\ University of Health and Allied Sciences \\ Gideon Kye-Duodu \\ University of Health and Allied Sciences \\ Francis Abeku Ussher \\ Koforidua Technical University \\ Thomas Boakye \\ University of Health and Allied Sciences \\ Daniel Adigbli \\ Krachi-West District Hospital \\ Louis Selasi Ameke \\ Ho Municipal Hospital \\ William Klutse Fianko \\ Hohoe Municopal Hospital \\ Robert Adedze-Kpodo \\ District Hospital, Sogakope \\ Henry Komla Letsa \\ Ho Municipal Hospital \\ Worlanyo Tashie \\ Ho Municipal Hospital \\ Noble Selorm Gbormittah \\ District Hospital, Sogakope \\ Godsway Edem Kpene \\ University of Health and Allied Sciences \\ James Osei-Yeboah \\ University of Health and Allied Sciences
}

Research article

Keywords: epidemiology, Treponemal Infection, public health, Ghana

Posted Date: August 14th, 2019

DOI: https://doi.org/10.21203/rs.2.9743/v2

License: (c) (i) This work is licensed under a Creative Commons Attribution 4.0 International License. Read Full License 


\section{Abstract}

Abstract Background: Treponemal infection is contagious and one of the oldest blood-borne infections, with great public health consequences. This study aimed to comparatively describe the five-year (2013-2017) regional epidemiology of Treponemal infection using pregnant women in the Sentinel Survey and apparently healthy blood donors as proxy for the general population at the four sentinel sites in the Volta Region of Ghana. Method: We analysed retrospectively data from 17,744 prospective blood donors aged between 18 to 58 years and 7,805 pregnant women in a Sentinel Survey who fell within the 15 and 49 years age bracket at Hohoe, Ho, Tongu and KrachiWest sentinel sites in the Volta Region. Data extracted included age, gender, date of blood donation and Treponena pallidum chromatographic immunoassay results from the blood banks of the four study sites. Published reports of Sentinel Surveys conducted at the four sentinel sites from the years 2013-2017 were retrieved. Results: The cumulative five-year prevalence of Treponemal infections among the pregnant women in the Sentinel Survey and prospective blood donors was $0.38 \%$ and $2.38 \%$ respectively. Site-specific prevalence for population-base/Sentinel survey was 4.6\%/0.4\%, 2.0\%/0.2\%, 1.3\%/0.8 and 1.2\%/0.2 for Hohoe, Ho, Krachi-West and Tongu respectively. Treponemal infection rates among the younger age groups (15-24years) were $0.31 \%$ in the sentinel survey and $2.22 \%$ in the general population. Significant gender disparity in Treponemal infection exist with male preponderance. Conclusion: The regional prevalence of Treponemal infection in the Sentinel Survey is lower compared to the general population. Therefore, the use of pregnant women as proxy for population estimate could lead to underestimation of the burden in the study jurisdiction.

\section{Introduction}

Treponematoses is one of the oldest bacterial diseases caused by the Spirochaete Treponema pallidum subspecies pallidum and pertenue and is a major public health problem worldwide [1]. The infection can be transmitted via transfusion of contaminated blood [2], vertically from mother to child and horizontal transmission through sexual intercourse with infected persons as well as through parenteral transmission among drug addicts [3]. Global reports indicate that Treponemal infection affects about 36 million people, with 12 million new cases reported every year [4]. In a recent WHO survey, about 1 million pregnant women were reported to have active Treponemal infection constituting a 1\% prevalence [5] while among blood donors, an estimated 1.6 million units of blood were discarded due to the presence of infectious markers including treponematoses [6]. In Africa, Treponemal infection in women of reproductive age group is reported to range from $0.36 \%$ to $3.6 \%$ [7] whereas rates ranging from $0.71 \%$ to $20 \%$ have been recorded among blood donors [8,9]. In Ghana, previous studies undertaken to establish national representative data of Treponemal infection among pregnant women and apparently healthy blood donors revealed prevalence rates of $0.3 \%$ and $3.7 \%$ among the respective populations [10, 11$]$. Mother-to-child transmission of Treponemal infection may lead to adverse pregnancy outcomes, including foetal loss, neonatal death, low birth weight, stillbirth and congenital abnormalities [12]. It is estimated that about 930,000 pregnant women experience nearly 350,000 adverse pregnancy outcomes yearly because of Treponemal infection [13]. Populations that are infected but show no signs and symptoms of the infection risk carrying the infection for a long period of time and if left untreated could serve as potential reservoirs for the spread of the infection [14]. There have been concerns about the representativeness of the utility of estimates from sentinel surveys using only pregnant women for the determination of population estimates of infectious diseases. This then called for the recommendation that periodic validations of ANC surveillance data are carried out through the conduct of population-based studies in countries with generalized epidemiology [15]. In pursuant to this recommendation, we previously described the epidemiology of Treponemal infection by comparing estimates from the sentinel survey with those observed among asymptomatic adult population using prospective blood donors as a proxy at the Ho Sentinel site in the Volta Region [16]. Findings from that study suggested that the use of only pregnant women might underestimate the population burden of the disease, laying credence to a possible unrepresentativeness of the use of Sentinel Surveys. Understanding the epidemiological pattern of Treponemal infection from a regional perspective could be an important step to reviewing national strategies aimed at eliminating the menace from the population. Using a similar approach, but a widened scope, the present study is therefore aimed at describing the five-year (2013-2017) regional epidemiology of Treponemal infection by comparing estimates from the sentinel survey and blood donors as proxy of the asymptomatic adult population at the four sentinel sites in the Volta Region of Ghana.

\section{Methods}

\subsection{Study Area and Study Sites}

The Volta Region is one of the ten administrative regions located at the eastern part of Ghana. It is bounded by the Northern Region to the north, south by the Gulf of Guinea, west by the Volta Lake and east by the Republic of Togo. The region occupies a surface area of about 20,570 square kilometres. The population of the region based on the national population and housing census in 2010 , was $1,901,179$, 
with an annual growth rate of $1.9 \%$. The largest populated district is Ho, with a population of 214,612 followed by Hohoe municipal with a population of 181,297 . The least populated district is Jasikan with a population of 58,483 .

\subsection{Study Design and Study Population}

This study is a retrospective analysis of secondary data from 17,744 prospective blood donors aged from 18 to 58 years and 7,805 pregnant women in a Sentinel Survey who fell within the 15 and 49 years age bracket. The study was conducted at the four HIV/STI sentinel sites in the Volta Region namely; Ho, Hohoe, Krachi-West and Tongu. Ho and Hohoe sites are located in urban Ghana, while Krachi-West and Tongu sites are of rural settings [17].

\subsection{Data Collection}

Data extracted included age, gender, date of donation and treponemal test results from the blood banks of the four study sites. All four sites employed chromatographic immunoassays for the qualitative detection of antibodies to Treponena palliium (TP) in human serum. In brief, two purified recombinant TP antigens are made into a test capture band material and a gold conjugate. The presence of $T P$ antibodies in a serum test sample forms a complex with the immobilized antigens in the test zone of the membrane leading to a visible pink-rose coloured band on the membrane.

Published reports of Treponemal infection from the National Sentinel Surveys conducted at the four sentinel sites spanning the period 2013-2017 were retrieved. The WHO standard algorithm for treponematoses screening in asymptomatic population recommends the utility of non treponemal based test-Rapid Plasma Reagin (RPR) and a treponemal test e.g. Treponema pallidum haemagglutination assay (TPHA) [18]. The RPR method is used to identify persons with possible untreated infections, followed by confirmation using a treponemal test e.g. TPHA [19]. However, results from the Sentinel Surveys were based on two sequential treponemal tests (Determine Syphilis TP and TPHA) [20] until 2015 where a combination of treponemal immunochromatographic and RPR testing was adopted.

\subsection{Data Analysis}

Data was collected, entered into Microsoft Excel 2016 spreadsheet and validated for entry errors. Data was presented as frequencies and proportions. Differences between proportions and trends analysis were carried out using Fisher's exact test, chi-square test and chi-square test for trend where appropriate. A p value $<0.05$ was considered as statistically significant. IBM Statistical Package for the Social Sciences (SPSS Inc. Chicago, USA; http://www.spss.com/) version 22.0 was used for analysis.

\subsection{Ethical Considerations}

Approval for this study was obtained from the authorities of the four sentinel hospitals. Ethical clearance for the study was obtained from the Research Ethics Committee of the University of Health and Allied Sciences, Ho (UHAS-REC A.4 [171] 18-19). The data retrieved were anonymous and non-linked. Patients' names and other attributes that could lead to identity disclosure were not retrieved from the archives.

\section{Results}

The cumulative five-year prevalence of Treponemal infections in the Volta Region was estimated as $0.38 \%$ by the Sentinel Survey, $2.38 \%$ infection rate was observed in the population based survey using all prospective blood donors and $2.31 \%$ infection rate among prospective donors within the Sentinel Survey age range (15-49). The year-on-year regional epidemiological pattern in the Sentinel Survey recorded a stable infection rate of under $1 \%$ for each year under review. With the exception of 2017, the estimated regional Treponemal infection in the population-based sampling was above $2 \%$ in each of the review years and statistically higher in comparison with what was reported in the Sentinel Survey. Table 1.

Table 1: Year-on-year prevalence of Treponemal infection among blood donors and pregnant women (Sentinel Survey) in the Volta Region 


\begin{tabular}{lccccccc}
\hline Parameter & Total & $\mathbf{2 0 1 3}$ & $\mathbf{2 0 1 4}$ & $\mathbf{2 0 1 5}$ & $\mathbf{2 0 1 6}$ & $\mathbf{2 0 1 7}$ & $\boldsymbol{p}$ for trends \\
\hline Sentinel & 7805 & 1287 & 1523 & 1719 & 1603 & 1673 & \\
Positive & $30(0.38)$ & $4(0.31)$ & $13(0.85)$ & $5(0.29)$ & $1(0.06)$ & $7(0.42)$ & 0.2191 \\
All donors & 17744 & 2753 & 3195 & 3336 & 4421 & 4039 & \\
Positive & $423(2.38)$ & $78(2.83)$ & $75(2.35)$ & $93(2.79)$ & $144(3.26)$ & $33(0.82)$ & 0.0001 \\
Donors (15/49) & 16858 & 2550 & 3036 & 3160 & 4250 & 3862 & \\
Positive & $389(2.31)$ & $69(2.71)$ & $70(2.31)$ & $86(2.72)$ & $131(3.08)$ & $33(0.85)$ & 0.0002 \\
$P$ & $<0.0001$ & $<0.0001$ & 0.0006 & $<0.0001$ & $<0.0001$ & 0.1416 & \\
$p 1$ & $<0.0001$ & $<0.0001$ & 0.0008 & $<0.0001$ & $<0.0001$ & 0.1127 & \\
\hline
\end{tabular}

Data is presented as frequency with the corresponding percentage in parenthesis. P compares all donors within the sentinel, p1 compares donor within 15-49 years with the sentinel. $\mathrm{P}$ is significant at 0.05.

The prevalence of Treponemal infections in the younger population aged between 15 and 24 years was $0.31 \%$ among the 2893 young participants who took part in the Sentinel Study in the Volta Region within the five years of the review. In the population-based study, 144 cases of Treponemal infections were recorded, representing $2.22 \%$ of the 6491 young participants. The proportion of Treponemal infection was found to be significantly higher in the population-based sample compared to the sentinel population, in each year during the first four years of the period under review (Table 2).

Table 2: Year-on-year prevalence of Treponemal infections among the younger population (15-24 years) of blood donors and pregnant women (Sentinel Survey) in the Volta Region

\begin{tabular}{|c|c|c|c|c|c|c|c|}
\hline Parameter & Total & 2013 & 2014 & 2015 & 2016 & 2017 & $p$ for trends \\
\hline Sentinel & 2893 & 505 & $\overline{584}$ & $\overline{614}$ & 577 & $\overline{613}$ & \\
\hline Positive & $9(0.31)$ & $2(0.40)$ & $4(0.68)$ & $1(0.16)$ & $0(0.00)$ & $2(0.33)$ & 0.2652 \\
\hline Donors & 6491 & 835 & 1345 & 1402 & 1747 & 1162 & \\
\hline Positive & $144(2.22)$ & $22(2.63)$ & $30(2.23)$ & $32(2.28)$ & $52(2.98)$ & $8(0.69)$ & 0.0558 \\
\hline$p$-value & $<0.0001$ & $<0.0022$ & 0.0220 & 0.0011 & $<0.0001$ & 0.5089 & \\
\hline
\end{tabular}

Data is presented as frequency with the corresponding percentage in parenthesis. $\mathrm{p}$ is significant at 0.05.

The epidemiological density of Treponemal infection among the sentinel population irrespective of age revealed a stable trend of less than $1 \%$, ranging from a low of $0 \%$ among the 15 to 19 and 45 to 49 year groups, and a high of $0.81 \%$ among the 40 to 44 year group. In the population-based sample the prevalence of Treponemal infection was above $2 \%$ for all age categories, except the trough age group (20 -24) years which recorded a rate of $1.85 \%$ (Table 3$)$.

Table 3: Five-year distribution of Treponemal infection by age categories 


\begin{tabular}{|c|c|c|c|c|c|c|c|c|c|c|}
\hline Parameter & $15-19$ & $20-24$ & $25-29$ & $30-34$ & 35-39 & $40-44$ & $45-49$ & $\geq 50$ & $\begin{array}{c}\text { Missing } \\
\text { Age }\end{array}$ & $\begin{array}{l}p \text { for } \\
\text { trends }\end{array}$ \\
\hline Sentinel & 983 & 1910 & 2142 & 1581 & 910 & 246 & 33 & - & - & \\
\hline Positive & $0(0.00)$ & $9(0.47)$ & $8(0.37)$ & $6(0.38)$ & $5(0.55)$ & $2(0.81)$ & $0(0.00)$ & - & - & 0.114 \\
\hline Donor & 1689 & 4802 & 4262 & 2653 & 1748 & 1070 & 634 & 525 & 361 & \\
\hline Positive & $55(3.26)$ & $89(1.85)$ & $92(2.16)$ & $63(2.37)$ & $40(2.29)$ & $30(2.80)$ & $20(3.15)$ & $13(2.48)$ & $21(2.82)$ & $<0.0001$ \\
\hline$p$-value & $\begin{array}{c}< \\
0.0001\end{array}$ & $\begin{array}{c}< \\
0.0001\end{array}$ & $\begin{array}{c}< \\
0.0001\end{array}$ & $\begin{array}{c}< \\
0.0001\end{array}$ & 0.0017 & 0.0688 & 0.6169 & nd & nd & \\
\hline
\end{tabular}

Data is presented as frequency with the corresponding percentage in parenthesis. $\mathrm{p}$ is significant at 0.05. nd: comparison analysis not done

In the multisite analysis, significant site specific differences in the cumulative Treponemal infection rate was observed $(p<0.0001)$. Hohoe with a prevalence of $4.6 \%$ recorded the highest burden of Treponemal infection within the five-year period of the review. Ho sentinel site was second $(2.02 \%)$ followed by Krachi West (1.34\%) and Tongu (1.18\%). With the exception of Tongu, which recorded the highest Treponemal infection in the year 2016 (2.08\%), the peak year of Treponemal infection was observed in the year 2013 for sites. The epidemiology pattern of Treponemal infection in general observed a decline at the end of the review year (2017) from the previous year (2016) in the four sites. However the margin of reduction in the case of Tongu was found to be statistically not significant $(p=0.5016)$. Table 4.

Table 4: Site specific year on year distribution of Treponemal infection among blood donors

\begin{tabular}{lccccccc}
\hline Parameter & Total & 2013 & 2014 & 2015 & 2016 & 2017 & $\begin{array}{c}\text { p for } \\
\text { trends }\end{array}$ \\
& & & & & & & \\
\hline Ho & $85 / 4212(2.02)$ & $32 / 1126(2.84)$ & $10 / 630(1.59)$ & $17 / 646(2.63)$ & $22 / 892(2.47)$ & $4 / 918(0.44)$ & 0.0031 \\
Tongu & $25 / 2127(1.18)$ & $2 / 225(0.89)$ & $1 / 300(0.33)$ & $2 / 464(0.43)$ & $11 / 529(2.08)$ & $9 / 609(1.48)$ & 0.0610 \\
& & & & & & & \\
Hohoe & $226 / 4908(4.60)$ & $18 / 273(6.59)$ & $48 / 1121(4.28)$ & $64 / 1122(5.70)$ & $87 / 1449(6.00)$ & $9 / 943(0.95)$ & 0.0013 \\
& & & & & & & \\
Krachi & $87 / 6497(1.34)$ & $26 / 1129(2.30)$ & $16 / 1144(1.40)$ & $10 / 1104(0.91)$ & $24 / 1551(1.55)$ & $11 / 1569(0.70)$ & 0.0030 \\
West & & & & & & & \\
p value & $<0.0001$ & 0.0004 & $<0.0001$ & $<0.0001$ & $<0.0001$ & 0.1442 &
\end{tabular}


Data is presented as frequency (positive/total) with the corresponding percentage in parenthesis. $\mathrm{p}$ is significant at 0.05. $\mathrm{p}$ for trends: evaluates significant yearly linear trends, $\mathrm{p}$ : inter-site comparison.

The five-year cumulative rate of Treponemal infection was found to be significantly higher in the population-based sample compared to the Sentinel Survey in all Volta Regional sentinel sites except Krachi West. The infection pattern in both populations at all Sentinel sites presented an undulating trend in the year-on-year Treponemal infection rate. Though not statistically significant in all cases, the population-based study presented a higher year-on-year Treponemal infection rate. However, the 2014 rate in Tongu and Krachi West bucked the trend with higher rates in the Sentinel Survey, while the same rate was recorded in both sample populations in 2015 at Krachi West (0.9\% each) (Figure 1D) and 2017 at Hohoe (1.0\% each) (Figure 1C). With the exception of Tongu which as of 2013 was not a sentinel site, the peak year in the population-based sample for all the other sites was at the beginning of the review period (2013) and troughed at the end of the review period (2017). Figure 1.

The cumulative prevalence of Treponemal infection was observed at $1.5 \%$ rate among the female population and $2.4 \%$ rate among their male counterparts, while the sentinel estimate was $0.4 \%$ (Figure $2 \mathrm{~A}$ ). The infection rate among the younger female subgroup (15-24years) was $1.6 \%, 2.3 \%$ among their male counterparts and $0.3 \%$ from the sentinel estimates (Figure 2B). There is a significant male preponderance in the cumulative five-year infection rate $(p=0.0433)$ (figure $2 A)$. However, no significant gender variation in the infection rate was observed among the younger populations of the two sample groups for the five-years under review (Figure 2B). The regional cumulative prevalence and the infection rate among the younger group of the population-based study were found to be significantly higher compared to the estimates from the Sentinel Survey irrespective of gender. As seen in figure 2A, the year-on-year estimates in both genders were found to be higher than rates from the Sentinel Survey. With the exception of year 2015 where the female estimate in the population-based sample was lower, the year-on-year rates of infection among the younger population was higher in both genders compared to the Sentinel Survey estimates. (Figure 2B). Moreover, there was a decline in the rate among the younger group in all subpopulations comparing the start of the review (2013) to the end (2015).

\section{Discussion}

To the best of our knowledge, this is the first comparative study aimed at describing the epidemiological pattern of Treponemal infection within a regional context taking into consideration the four HIV/STI sentinel sites (Ho, Hohoe, Tongu and Krachi-West) in the Volta Region. The cumulative rate of Treponemal infection over the five-year review period (2013-2017) was $0.38 \%$ among the 7,805 pregnant women in the Sentinel Survey and $2.38 \%$ in the population-based survey among the 17,744 apparently healthy adult population. The five-year cumulative rate observed among the 16,858 asymptomatic adult population who fell below 50 years of age was $2.31 \%$ (Table 1). The infection rates recorded in this study are lower compared to the 5.1\% reported in Southern Ethiopia [21], 3.7\% in Gondar, Ethiopia [22], 5.1\% in Uganda [23], 3.0\% in Madagascar [24] and 7.2\% in Tanzania [25]. However, similar reports have been observed in other jurisdictions including 1.1\% in Ethiopia [26], 0.5\% in South Africa [27], $1.1 \%$ in Nigeria [28] and 2.9\% in Hungary [29]. Although we recorded a relatively low infection rate in this study in comparison to other settings, our findings suggest that Treponemal infection is persistent in the population and this constitutes an important public health issue in the current study jurisdiction. The phenomenon poses a challenge to WHO's efforts in achieving total elimination of congenital transmission of Treponemal infection by 2015 [30].

In general, the cumulative and yearly prevalence of Treponemal infection were significantly higher in the population-based group as compared to that recorded in the Sentinel Survey $(p<0.05)$, except for year 2017 where the difference was comparable between the two groups $(p=0.1416)$ (Table 1). Thus, our earlier finding of a difference in the rate of Treponemal infections between the two sample groups was not limited to the Ho sentinel site [16], but represents a regional picture. Earlier reports in other African countries also corroborated the results of this study. Studies in Tanzania [31-33], Uganda [34] and Zambia [35] reported that sexually transmitted pathogens were 10-30\% lower in pregnant women in the Sentinel Survey compared to individuals in the general population. The finding of a lower rate of Treponemal infection in the Sentinel Survey compared to the population based group [16] raises important questions about the representativeness of the use of pregnant women for population estimates in the study jurisdiction. According to Boisson, et al. [36] and Zaba and Gregson [37], biases exist for using pregnant women as proxy for estimating the prevalence of sexually transmitted pathogens. In the opinion of Gregson, et al. [38], not all pregnant women would attend antenatal clinic, and attendance may also differ by socioeconomic status, age, locality, educational status, parity, ethnic group and religion. Moreover, the use of pregnant women for population estimates may not account for the burden in non-pregnant women of similar reproductive age bracket as well as men in the general population [39]. The reason for the significant reduction in the proportion of Treponemal infection (3.08\%) in 2016 to $0.85 \% 2017$ in the donor population within a year is unclear from this study. However, this may be due to the selection criteria or testing effect. 
In the present study, there was generally an increasing rate of Treponemal infection with increasing age in both the sentinel survey and population based group (Table 3). The findings are in concordance with those reported by Endris, et al. [40] in Ethiopia, Pham, et al. [41] in Zimbabwe, Yang, et al. [42] in China and Noyola, et al. [43] in Mexico. The possible explanation to the high prevalence of Treponemal infection among the older population in this study could be due to the less common use of condom and keeping multiple sexual partners which could predispose them to a higher risk of infection. This view was also corroborated by Endris, et al. [40]. In this study, we recorded Treponemal infection rates of $0.31 \%$ and $2.22 \%$ among the younger group in the sentinel survey and the general population respectively. Though the rate is lower compared to the older population, it suggests that Treponemal infection also exists among the younger generation in the study region. Hence, this calls for the up-scaling of activities aimed at reducing the infection rate among the younger population through early identification and treatment to break the spread from affected persons, and discouraging the youth from engaging in unprotected sexual intercourse with multiple partners and education among others.

In the multisite analysis, we found the cumulative Treponemal infection rate to be highest in Hohoe (4.6\%), followed by Ho (2.02\%), Krachi-West (1.34\%) and Tongu (1.18\%) during the five-year period under review (Table 4). The cumulative prevalence of Treponemal infection was significantly higher in the population-based sample compared to the Sentinel Survey in all the sentinel sites except KrachiWest, whiles the yearly infection pattern in both populations at all the sentinel sites presented with a characteristic undulating trend (Figures 1A, 1B, 1C, and 1D). Hohoe and Ho sentinel sites are located within urban Ghana whiles Krachi-West and Tongu sites are of rural origin [17]. There is a strong and consistent link between conditions prevalent in urban areas and the spread of sexually transmitted infections. Factors including urban poverty, resulting from inequalities in socio-economic status among dwellers could lead to the dependence of girls and women on men who are gainfully employed for economic survival resulting in high rates of unintended pregnancies and sexually transmitted infections [44].

In the present study, we observed a significant gender disparity of Treponemal infection for the five year under review. The male subpopulation recorded higher Treponemal infection (2.4\%) compared to their female counterparts $(1.5 \%)(p=0.0433)$ (Figure 2A). Though not statistically significant, the infection rate among the younger male subpopulation was higher than their female peers (Figure 2B). In our previous study, we have observed male preponderance to Treponemal infection [16], and also in the works of Anwar, et al. [45]. On the contrary, Coffin, et al. [46] and Gao, et al. [47], opined that females were more susceptible to Treponemal infection than their male counterparts. The higher infection rate observed among the male subpopulation could possibly be due in part to the disproportionate gender distribution of the donor participants which was skewed towards the male population.

The present study suffers from some limitations worth mentioning, as treponematoses endemicity of study area, choice of testing method and difference in STI risk exposures (pregnant women are an HIV/STI risk population due to exposure to unprotected sex, while the STI risk of donor population is unknown) are crucial for results interpretation. This study employed retrospective data on treponemal test results from donor population and published data of HSS results of syphilis surveillance among pregnant women. Treponema antibodies may persist for life whether an infection is treated or not, whereas RPR decreases in titre and could vanish when successful treatment is given [18]. Since RPR testing was not performed for the donor population and Sentinel Survey until 2015, as well as, given the fact that the study jurisdiction is known to be yaws endemic [48], it is possible that the results from this study could be overestimated. This is because immunochromatographic based testing method is unable to differentiate between the causative organism for yaws (Treponema pertenue) and Treponema pallidium. A previous study undertaken by Dassah, et al. [48] which involved retesting of archived 2007 HSS samples of Ghanaians found that over $50 \%$ of samples considered to have "active infection" using the HSS strategy were classified as past or treated infections based on the WHO recommended algorithm.

\section{Conclusion}

The regional prevalence of Treponemal infection in the Sentinel Survey is lower compared to the general population. Therefore, the use of pregnant women as proxy for population estimates could lead to underestimation in the study jurisdiction.

\section{Declarations}

\section{Acknowledgement}

We wish to thank the management of the four hospitals (sentinel sites) used in our study.

\section{Conflict of Interest}

Authors declare there is no conflict of interest 


\section{Funding}

No funding was received for this study.

\section{Data availability}

The datasets used during the current study are available from the corresponding author on request.

\section{Author's contribution}

This work was carried out in collaboration among all authors. All authors have read and reviewed the content of the final manuscript and have approved the manuscript for submission

\section{References}

1. T. Shimelis, K. Lemma, H. Ambachew and E. Tadesse, "Syphilis among people with HIV infection in southern Ethiopia: seroprevalence and risk factors," BMC infectious diseases, vol. 15, no. 1, pp. 189, 2015.

2. L. Vera, D. Milka, S.-L. Nurith and S. Eilat, "Prevalence and incidence of syphilis among volunteer blood donors in Israel," Journal of blood transfusion, vol. 2014, 2014.

3. O. Loza, T. L. Patterson, M. Rusch, G. A. Martínez, R. Lozada, H. Staines-Orozco, C. Magis-Rodríguez and S. A. Strathdee, "Drug-related behaviors independently associated with syphilis infection among female sex workers in two Mexico-US border cities," Addiction, vol. 105, no. 8, pp. 1448-1456, 2010.

4. G. Elyamany, "Prevalence of Syphilis among Blood and Stem Cell Donors in Saudi Arabia: An Institutional Experience," Electronic physician, vol. 8, no. 8, pp. 2747, 2016.

5. WHO, "Global Health Observatory (GHO) data-Sexually Transmitted Infections (STIs) ", Ed., 2018.

6. WHO, "Blood Donor Selection-Guidelines on Assessing Donor Suitability for Blood Donation ", Ed., 2012.

7. E. L. Korenromp, G. Mahiané, J. Rowley, N. Nagelkerke, L. Abu-Raddad, F. Ndowa, A. El-Kettani, H. El-Rhilani, P. Mayaud and R. M. Chico, "Estimating prevalence trends in adult gonorrhoea and syphilis in low-and middle-income countries with the Spectrum-STI model: results for Zimbabwe and Morocco from 1995 to 2016," Sex Transm Infect, pp. sextrans-2016-052953, 2017.

8. T. Deressa, W. Birhan, B. Enawgaw, M. Abebe, H. W. Baynes, M. Desta, B. Terefe and M. Melku, "Proportion and predictors of transfusion-transmissible infections among blood donors in North Shewa Zone, Central North Ethiopia," PloS one, vol. 13, no. 3, pp. e0194083, 2018.

9. E. Quintas, A. D. C. Cogle, A. Sebastião, A. da Costa Pereira, A. Sarmento, J. Van-Dúnem and L. Cordeiro, "Prevalence of syphilis in blood donors in angola from 2011 to 2016," 2018.

10. F. Sarkodie, O. Hassall, E. Owusu-Dabo, S. Owusu-Ofori, I. Bates, I. C. Bygbjerg, J. K. Ansah and H. Ullum, "Syphilis screening practices in blood transfusion facilities in Ghana," International Journal of Infectious Diseases, vol. 43, pp. 90-94, 2016.

11. NACP, "2017 HIV Sentinel Reports," Ed., 2018.

12. G. B. Gomez, M. L. Kamb, L. M. Newman, J. Mark, N. Broutet and S. J. Hawkes, "Untreated maternal syphilis and adverse outcomes of pregnancy: a systematic review and meta-analysis," Bulletin of the World Health Organization, vol. 91, pp. 217-226, 2013.

13. N. S. Wijesooriya, R. W. Rochat, M. L. Kamb, P. Turlapati, M. Temmerman, N. Broutet and L. M. Newman, "Global burden of maternal and congenital syphilis in 2008 and 2012: a health systems modelling study," The Lancet Global health, vol. 4, no. 8, pp. e525-e533, 2016.

14. CDC, "Sexually Transmitted Disease Surveillance 2017," Ed., 2018.

15. JUNP/WHO, "Guidelines for conducting HIV sentinel serosurveys among pregnant women and other groups," Ed., 2003.

16. J. Osei-Yeboah, S. Y. Lokpo, F. A. Ussher, V. N. Orish, A.-W. M. Hamid, M. P. Dakorah, T. Ntoni, E. A. Nani, F. Ayroe and D. Adigbli, "The Epidemiology of Human Immunodeficiency Virus (HIV) and Syphilis in Ghana: A Five-Year Single Urban Site Parallel PopulationBased Analysis vis-à-vis the Sentinel Survey," Journal of Tropical Medicine, vol. 2018, 2018.

17. GAC, "Summary of the 2017 HIV Sentinel Survey Report, Ghana AIDS Commission, Ghana," Ed., 2018.

18. R. W. Peeling and H. Ye, "Diagnostic tools for preventing and managing maternal and congenital syphilis: an overview," Bulletin of the World Health Organization, vol. 82, pp. 439-446, 2004. 
19. CDC and Prevention, "Discordant results from reverse sequence syphilis screening-five laboratories, United States, 2006-2010," MMWR. Morbidity and mortality weekly report, vol. 60, no. 5, pp. 133, 2011.

20. NACP/GHS, "2010 HIV sentinel survey report. Accra," Ed., 2010.

21. A. Amsalu, G. Ferede and D. Assegu, "High seroprevalence of syphilis infection among pregnant women in Yiregalem hospital southern Ethiopia," BMC infectious diseases, vol. 18, no. 1, pp. 109, 2018.

22. M. Melku, A. Kebede and Z. Addis, "Magnitude of HIV and syphilis seroprevalence among pregnant women in gondar, northwest ethiopia: a cross-sectional study," HIV/AIDS (Auckland, NZ), vol. 7, pp. 175, 2015.

23. Y. C. Manabe, G. Namale, E. Nalintya, J. Sempa, R. P. Ratanshi, N. Pakker and E. Katabira, "Integration of antenatal syphilis screening in an urban HIV clinic: a feasibility study," BMC infectious diseases, vol. 15, no. 1, pp. 15, 2015.

24. H. Frickmann, N. G. Schwarz, M. Girmann, R. M. Hagen, S. Poppert, S. Crusius, A. Podbielski, J. N. Heriniaina, T. Razafindrabe and J. P. Rakotondrainiarivelo, "Serological survey of HIV and syphilis in pregnant women in Madagascar," Tropical Medicine \& International Health, vol. 18, no. 1, pp. 35-39, 2013.

25. R. O. Swai, M. I. Matee, J. Killewo, E. F. Lyamuya, G. Kwesigabo, T. Tulli, T. K. Kabalimu, R. Isingo and J. Ndayongeje, "Surveillance of HIV and syphilis infections among antenatal clinic attendees in Tanzania-2003/2004," BMC Public Health, vol. 6, no. 1, pp. 91, 2006.

26. D. Kassa, G. Gebremichael, T. Tilahun, A. Ayalkebet, Y. Abrha, G. Mesfin, Y. Belay, M. Demissie, A. Gebrexiabher and Y. Assefa, "Prevalence of sexually transmitted infections (HIV, hepatitis B virus, herpes simplex virus type 2, and syphilis) in pregnant women in Ethiopia: Trends over 10 years (2005-2014)," International Journal of Infectious Diseases, vol. 79, pp. 50-57, 2019.

27. R. S. Kularatne, R. Niit, J. Rowley, T. Kufa-Chakezha, R. P. Peters, M. M. Taylor, L. F. Johnson and E. L. Korenromp, "Adult gonorrhea, chlamydia and syphilis prevalence, incidence, treatment and syndromic case reporting in South Africa: Estimates using the Spectrum-STI model, 1990-2017," PloS one, vol. 13, no. 10, pp. e0205863, 2018.

28. F. Magaji, A. Ocheke, V. Pam, T. Afolaramin, J. Musa, A. Sagay and A. Zoakah, "Coverage and Uptake of Syphilis Screening among Pregnant Women Attending Antenatal Care: A 5-Year Review in Plateau State, Nigeria," International Journal of Advanced Research in Gynaecology and Obstetrics, vol. 1, no. 1, pp. 6-10, 2018.

29. E. Balla and G. G. Donders, "Features of syphilis seropositive pregnant women raising alarms in Hungary, 2013-2016," European Journal of Obstetrics \& Gynecology and Reproductive Biology, vol. 228, pp. 274-278, 2018.

30. WHO, "The global elimination of congenital syphilis: rationale and strategy for action; 2007," Ed., 2007.

31. M. Borgdorff, L. Barongo, A. Klokke, K. Senkoro, J. Newell, A. Nicoll, F. Mosha, H. Grosskurth and R. Swai, "Sentinel surveillance for HIV-1 infection: how representative are blood donors, outpatients with fever, anaemia, or sexually transmitted diseases, and antenatal clinic attenders in Mwanza Region, Tanzania?," AIDS (London, England), vol. 7, no. 4, pp. 567-572, 1993.

32. G. Kwesigabo, J. Z. Killewo, W. Urassa, E. Mbena, F. Mhalu, J. Lugalla, C. Godoy, G. Biberfeld, M. Emmelin and S. Wall, "Monitoring of HIV-1 infection prevalence and trends in the general population using pregnant women as a sentinel population: 9 years experience from the Kagera region of Tanzania," Journal of acquired immune deficiency syndromes (1999), vol. 23, no. 5, pp. 410-417, 2000.

33. J. Changalucha, H. Grosskurth, W. Mwita, J. Todd, D. Ross, P. Mayaud, A. Mahamoud, A. Klokke, F. Mosha and R. Hayes, "Comparison of HIV prevalences in community-based and antenatal clinic surveys in rural Mwanza, Tanzania," Aids, vol. 16, no. 4, pp. 661-665, 2002.

34. A. H. Kilian, S. Gregson, B. Ndyanabangi, K. Walusaga, W. Kipp, G. Sahlmüller, G. P. Garnett, G. Asiimwe-Okiror, G. Kabagambe and P. Weis, "Reductions in risk behaviour provide the most consistent explanation for declining HIV-1 prevalence in Uganda," Aids, vol. 13, no. 3, pp. 391-398, 1999.

35. K. Fylkesnes, Z. Ndhlovu, K. Kasumba, R. M. Musonda and M. Sichone, "Studying dynamics of the HIV epidemic: population-based data compared with sentinel surveillance in Zambia," Aids, vol. 12, no. 10, pp. 1227-1242, 1998.

36. E. Boisson, A. Nicoll, B. Zaba and L. C. Rodrigues, "Interpreting HIV seroprevalence data from pregnant women," JAIDS Journal of Acquired Immune Deficiency Syndromes, vol. 13, no. 5, pp. 434-439, 1996.

37. B. Zaba and S. Gregson, "Measuring the impact of HIV on fertility in Africa," AIDS (London, England), vol. 12, pp. S41-50, 1998.

38. S. Gregson, T. Zhuwau, R. M. Anderson, T. Chimbadzwa and S. K. Chiwandiwa, "Age and religion selection biases in HIV-1 prevalence data from antenatal clinics in Manicaland, Zimbabwe," The Central African journal of medicine, vol. 41, no. 11, pp. 339-346, 1995.

39. E. Gonese, J. Dzangare, S. Gregson, N. Jonga, O. Mugurungi and V. Mishra, "Comparison of HIV prevalence estimates for Zimbabwe from antenatal clinic surveillance (2006) and the 2005-06 Zimbabwe Demographic and Health Survey," PLoS one, vol. 5, no. 11, pp. e13819, 2010. 
40. M. Endris, T. Deressa, Y. Belyhun and F. Moges, "Seroprevalence of syphilis and human immunodeficiency virus infections among pregnant women who attend the University of Gondar teaching hospital, Northwest Ethiopia: a cross sectional study," BMC infectious diseases, vol. 15, no. 1, pp. 111, 2015.

41. L. Pham, G. Woelk, Y. Ning, S. Madzime, S. Mudzamiri, K. Mahomed and M. Williams, "Seroprevalence and risk factors of syphilis infection in pregnant women delivering at Harare Maternity Hospital, Zimbabwe," The Central African journal of medicine, vol. 51, no. 3-4, pp. 24-30, 2005.

42. L.-G. Yang, J. D. Tucker, F.-Y. Liu, X.-Q. Ren, X. Hong, C. Wang, M. M. McLaughlin, C. H. Bien, X.-S. Chen and B. Yang, "Syphilis screening among 27,150 pregnant women in South Chinese rural areas using point-of-care tests," PloS one, vol. 8, no. 8, pp. e72149, 2013.

43. D. E. Noyola, O. Malacara-Alfaro, V. Lima-Rogel and A. Torres-Montes, "Seroprevalence of syphilis in pregnant women in San Luis Potosí," Salud publica de Mexico, vol. 48, no. 2, pp. 151-154, 2006.

44. E. Maclean, "Sex and the Cities: The Impact of Urbanisation on Sexually Transmitted Disease," Ed., 2016.

45. F. Anwar, I. A. Shah and M. Zeeshan, "Prevalence of Syphilis in Blood Donors in District Mardan Khyber Pakhtunkhwa Pakistan," International Journal of Contemporary Research and Review, vol. 9, no. 07, pp. 20257-20261, 2018.

46. L. S. Coffin, A. Newberry, H. Hagan, C. M. Cleland, D. C. Des Jarlais and D. C. Perlman, "Syphilis in drug users in low and middle income countries," International Journal of Drug Policy, vol. 21, no. 1, pp. 20-27, 2010.

47. J. Gao, J. Xu, Y. Sheng, X. Zhang, C. Zhang, Y. Li, B. Liang, L. Sun, S. Yang and X. Zhang, "Increasing trend of syphilis and infection resistance: a retrospective study," International Journal of Infectious Diseases, vol. 17, no. 11, pp. e971-e976, 2013.

48. E. T. Dassah, Y. Adu-Sarkodie and P. Mayaud, "Performance of Syphilis Sentinel Surveillance in the context of endemic Treponematoses: experience from Ghana," BMC infectious diseases, vol. 16, no. 1, pp. 745, 2016.

\section{Figures}
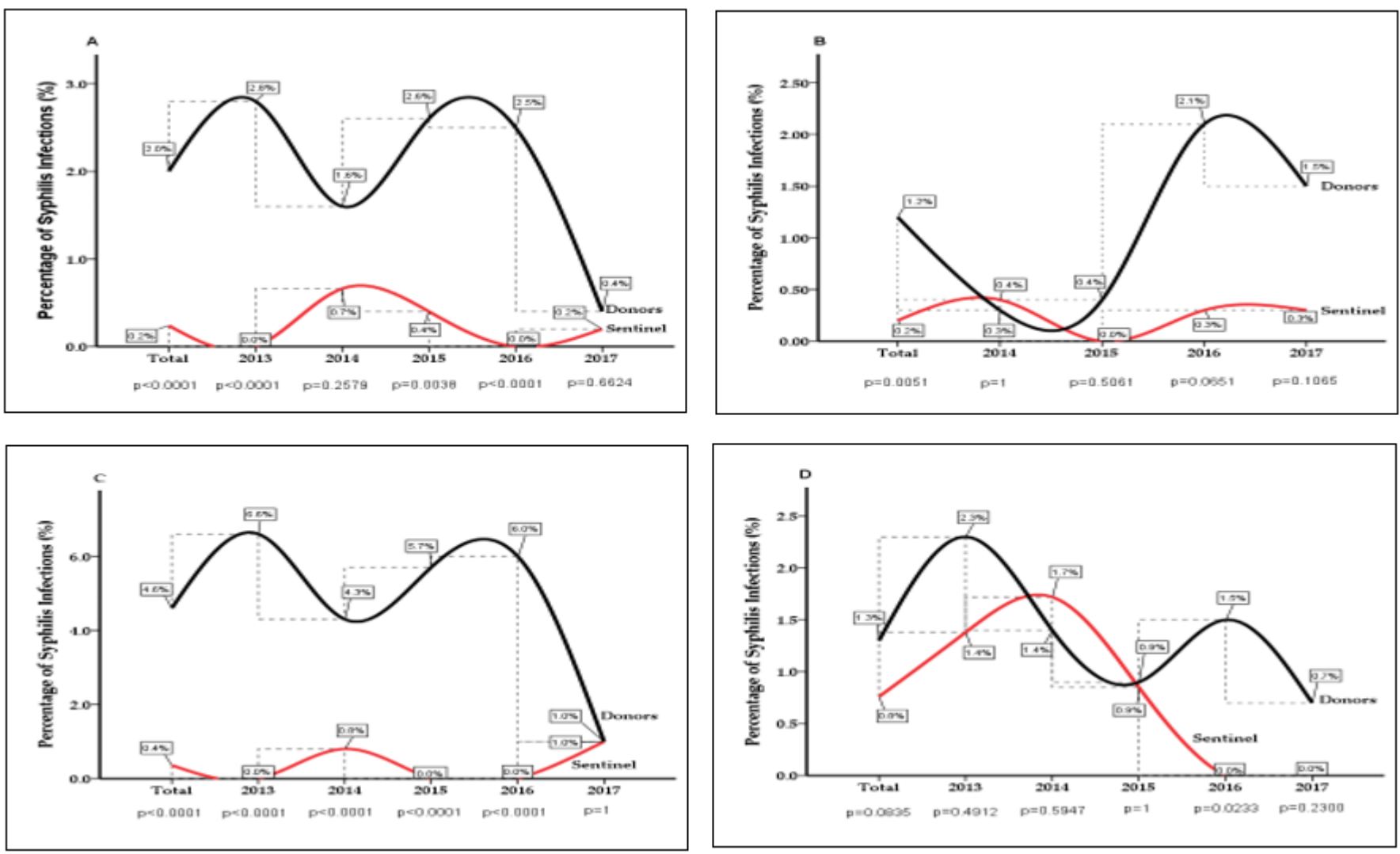

\section{Figure 1}

Year-on-year site specific Treponemal infection prevalence parallel analysis with the Sentinel Survey at the four Volta Region Sentinel sites. A: Ho Sentinel Site, B: Tongu Sentinel Site, C: Hohoe Sentinel Site and D: Krachi West Sentinel Site. p is significant at 0.05 

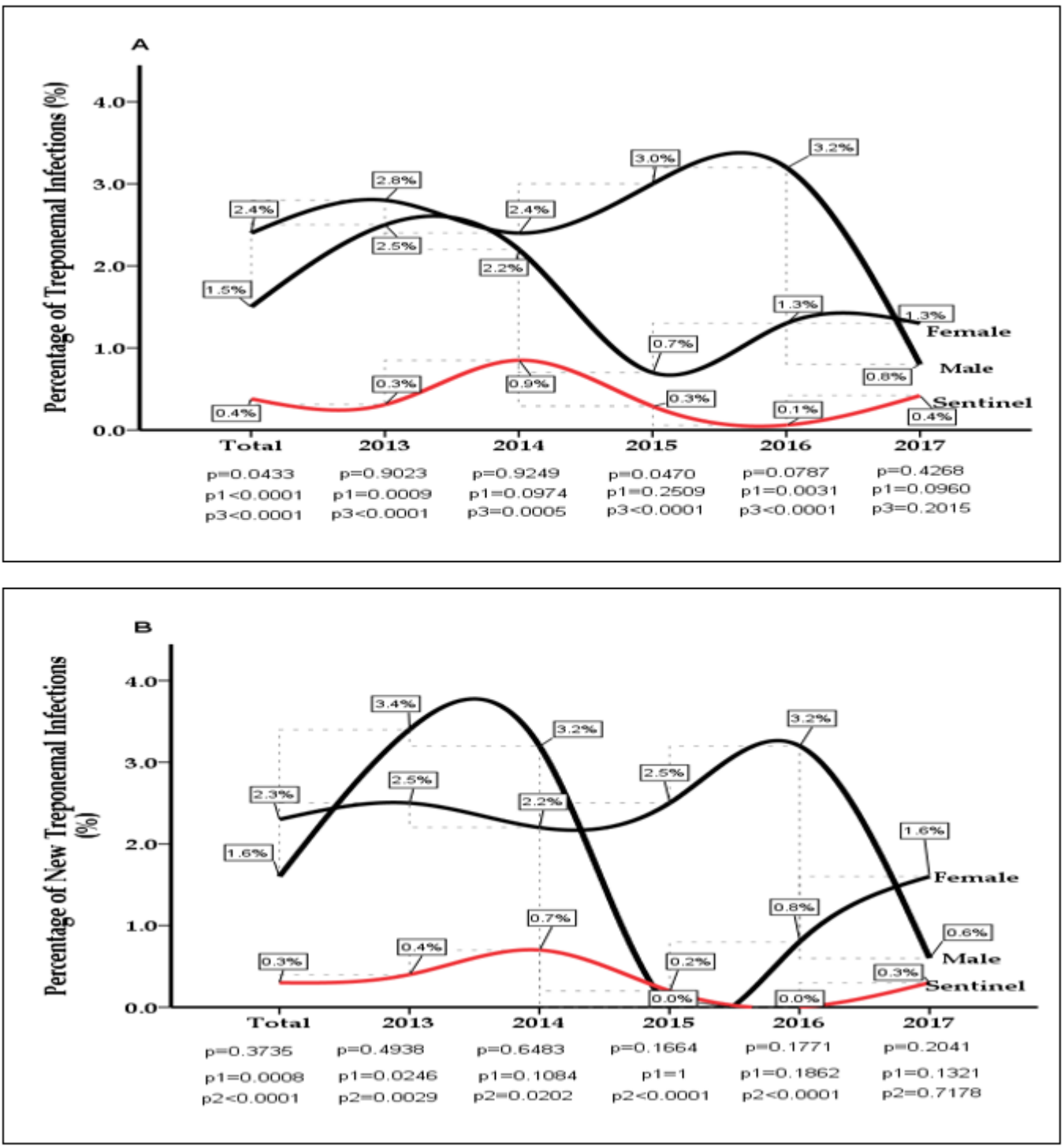

Figure 2

Year-on-year gender specific prevalence of Treponemal infection parallel analysis with the Sentinel Survey in the Volta Region. A: All Donors, B: 15-49years Donors. p is significant at 0.05 